\title{
AI Flame Detection System by Infrared Thermography
}

\author{
Man Yong Choi/ KRISS \\ mychoi@kriss.re.kr
}

\author{
Su Un Kim/Han Sun Co. Korea/co-author
}

\begin{abstract}
It is a very rapid spread that ends all situations in less than three minutes in many cases of fire, the current flame detector is slow to detect initial fire, which sometimes has a false alarm rate without general solution in the past decade. Artificial intelligent flame detector was designed using machine learning technology based on infrared thermal sensor, and intelligent flame detection technology was developed to accurately detect fire within few seconds of the initial flame stage. In order to improve reliability of AI flame detection, we minmized false alarm rate by distinguishing between fire and actual fire used by humans, and it is an intelligent fire recognition and response system technology of an architecture. The flame detection range is $50 \mathrm{~m}$ and response time is $3 \sim 5 \mathrm{sec}$.
\end{abstract}

